\title{
A Solution of Permutation Problem Inherent in Frequency Domain ICA
}

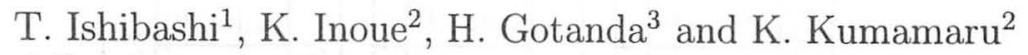 \\ 1 Graduate School of Computer Science and Systems Engineering, Kyushu Institute of Technology \\ 2 Faculty of Computer Science and Systems Engineering, Kyushu Institute of Technology \\ 680-4 Kawazu, Iizuka-shi, Fukuoka, 820-8502, JAPAN \\ 3 Faculty of Humanity-Oriented Science and Engineering, Kinki University \\ 11-6 Kayanomori, Iizuka-shi, Fukuoka 820-8555, JAPAN \\ E-mail: takaaki@matsu.ces.kyutech.ac.jp
}

\begin{abstract}
Under a multi-source and multi-microphone configuration, a new method is proposed for solving the permutation problem inherent in frequency domain independent component analysis (ICA) by utilizing prior informatin about the relative position of sound sources. The proposed method can also solve the channel selection problem for selecting the only channel for the target sound estimate, which is critical to a practical application of ICA.
\end{abstract}

\section{Introduction}

Recently, the speech recognition technology has significantly been improved to achieve provision of speech recognition engine with extremely high recognition capabilities for the case of ideal environments, i.e. no surrounding noise. However, it is still difficult to attain a desirable recognition rate in a household or office where there are daily activities noises. Therefore, a certain preprocessing before recognition is needed to reduce the noises and to select the target speech signal.

Many noise reduction methods using ICA $[1,2,3$, $4,5,6,7]$ have been proposed. ICA can separate unknown sources from their mixtures without information on the transfer functions, provided that the sources are statistically independent. For the instantaneous mixtures, the original sources can be completely recovered in the time domain except for indeterminacy of scaling and permutation. In a real environment, however, the signals observed at microphones are not instantaneous mixtures but are convoluted version of the sound sources. On account of this, there have been reported many trials to separate the convoluted mixtures in the frequency domain. However, the indeterminacy of scaling and permutation appears at every frequency bin. In order to recover the sources properly, this indeterminacy problem must be essentially solved before making an inverse transformation from the frequency to the time domain.
In order to solve the problem, several separation methods have been proposed. Scaling indeterminacy, i.e. amplitude and phase indeterminacy can be solved using decomposed spectrum [7] introduced by Murata et al. For the permutation problem, there have been tried a method using similarities between separate spectra [7], a method substituting the initial weights at a frequency by those learned at its adjacent frequency [8], a method taking advantage of directivity of arraymicrophones $[9,10]$ and a method utilizing the fact that entropy of human speech is in general lower than that of noise [11]. The last method is superior to the others but is not able to be applied to the case where an interference noise is a human utterance. We have proposed a permutation correction using prior information about source location under a two-source and two-microphone configuration [12]. We have also proposed to extract only the desired speech [12], making the best use of the FastICA's property that it separates the signal in order of large non-Gaussianity $[5,6]$.

The present paper shows that our method can be easily extended to the cases of a multi-source and multimicrophone configuration. Section 2 outlines the blind source separation and the FastICA algorithm in the frequency domain. Scaling and permutation problem is described. It is clarified that the decomposed spectrum is uniquely expressed in terms of the product of a source and its transfer function to the corresponding microphone. This fact settles the scaling indeterminacy. In Section 3, a solution of permutation ploblem is proposed under a multi-source and multi-microphone configuration using relative location on the sources. $\mathrm{Hu}-$ man speech extraction is also proposed. In section 4, from the results of several experiments it is confirmed that the proposed correction works robustly not only in a stationary noise but also in a non-stationary noise, and that a target speech can be surely extracted from the proposed extraction. Section 5 concludes the paper. 


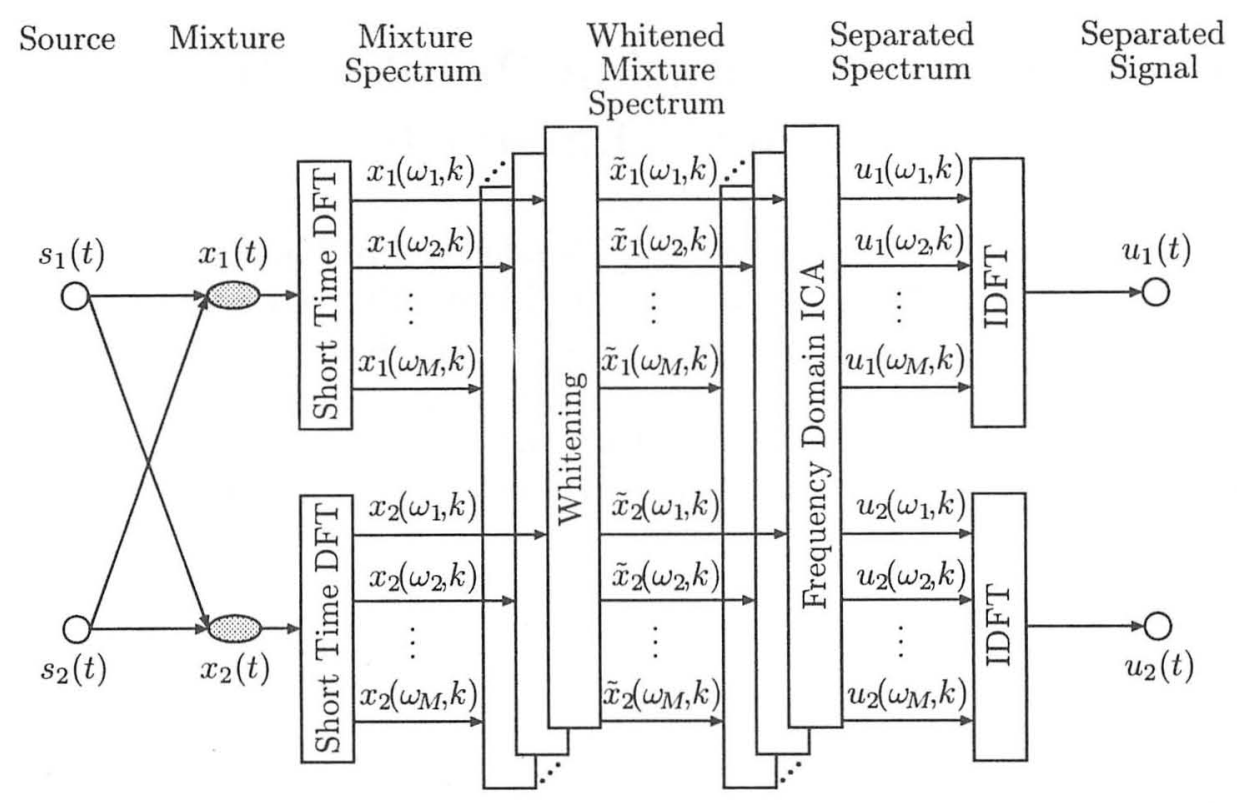

Fig. 1: Frequency domain ICA

\section{Blind Source Separation}

\subsection{Frequency Domain ICA}

The source signals are denoted by a vector

$$
s(t)=\left[s_{1}(t), \cdots, s_{I}(t)\right]^{T}
$$

where the components of $s(t)$ are statistically independent. Consider the case where sources are observed by microphones as mixture signals $x(t)=$ $\left[x_{1}(t), \cdots, x_{J}(t)\right]^{T}$ in a convoluted way:

$$
x(t)=G(t) * s(t)
$$

where $G(t)$ denotes a mixing matrix whose elements are transfer functions from the sources to the microphones, and $*$ denotes the convolutional operator.

The mixtures are transformed into the short time spectra by the discrete Fourier transform to perform separation in frequency domain.

$$
x_{j}(\omega, k)=\sum_{t} e^{-\sqrt{-1} \omega t} x_{j}(t) w(t-k \tau)
$$

where $\omega(=0,2 \pi / M, \cdots, 2 \pi(M-1) / M)$ denotes a frequency bin, $M$ the number of the samples in a frame, $k$ the frame number, $\tau$ the frame shift time and $w(t)$ a window function.

In the frequency domain, the mixtures $x(\omega, k)=$ $\left[x_{1}(\omega, k), \cdots, x_{J}(\omega, k)\right]$ are approximated as

$$
x(\omega, k)=G(\omega) s(\omega, k)
$$

where $s(\omega, k)=\left[s_{1}(\omega, k), \cdots, s_{I}(\omega, k)\right]$ and $G(\omega)$ are the discrete Fourier transformed representation of the windowed sources and the transfer function matrix, respectively. The mixtures are generally whitened by

$$
\widetilde{x}(\omega, k)=Q(\omega) x(\omega, k)
$$

where $Q(\omega)$ is a whitening matrix. The separated spectra $\boldsymbol{u}(\omega, k)=\left[u_{1}(\omega, k), \cdots, u_{N}(\omega, k)\right]^{T}$ can be obtained as

$$
u(\omega, k)=H(\omega) \boldsymbol{x}(\omega, k)
$$

where $H(\omega)=\left[\overline{\boldsymbol{h}}_{1}(\omega), \cdots, \overline{\boldsymbol{h}}_{N}(\omega)\right]^{T}$ is a demixing matrix, ${ }^{-}$a conjugation.

The separated signal $u_{n}(t)$ in the time domain for the source $s_{i}(t)$ can be obtained by applying the inverse Fourier transform of spectrograms $\left\{u_{n}(\omega, k) \mid k=\right.$ $0,1, \cdots, K-1\}$.

$$
u_{n}(t)=\frac{1}{2 \pi} \frac{1}{W(t)} \sum_{k} \sum_{\omega} e^{\sqrt{-1} \omega(t-k \tau)} u_{n}(\omega, k)
$$

where $W(t)=\sum_{k} w(t-k \tau)$. Frequency domain ICA under the two-source and two-microphone configuration is shown in Fig.1.

\subsection{FastICA in Frequency Domain}

Here we assume that all the spectra of whitened mixtures $\tilde{x}$ are zero mean and unit variance with uncorrelated real and imaginary parts of an equal variance. The FastICA algorithm is formulated in the frequency domain as follows [6].

$$
\begin{aligned}
h_{n}^{+}(\omega)= & \frac{1}{K} \sum_{k=0}^{K-1}\left\{\widetilde{\boldsymbol{x}}(\omega, k) \overline{u_{n}}(\omega, k) f\left(\left|u_{n}(\omega, k)\right|^{2}\right)\right. \\
& -\left[f\left(\left|u_{n}(\omega, k)\right|^{2}\right)\right. \\
& \left.\left.+\left|u_{n}(\omega, k)\right|^{2} f^{\prime}\left(\left|u_{n}(\omega, k)\right|^{2}\right)\right] \boldsymbol{h}_{n}(\omega)\right\} \\
\boldsymbol{h}_{n}(\omega)= & \frac{\boldsymbol{h}_{n}^{+}(\omega)}{\left\|\boldsymbol{h}_{n}^{+}(\omega)\right\|}
\end{aligned}
$$


where $h_{n}(\omega)$ is a demixing weight vector, $f(\cdot)$ a nonlinear function and $f^{\prime}(\cdot)$ is its differential.

At each frequency $\omega$, the convergence condition is

$$
\left|\bar{h}_{n, \text { old }}^{T}(\omega) \boldsymbol{h}_{n, \text { new }}(\omega)\right| \simeq 1
$$

where the subscript old and new denote the demixing weight before and that after update, respectively.

In addition, $\boldsymbol{h}_{n+1}(\omega)$ is orthogonalized by

$$
h_{n+1}(\omega)=h_{n+1}(\omega)-\sum_{j=1}^{n} h_{j}(\omega) \bar{h}_{j}^{T}(\omega) h_{n+1}(\omega)
$$

and $h_{n+1}(\omega)$ is again regularized by Eq.(9).

The separated spectra $u_{n}(\omega, k)$ are yielded by substituting $\boldsymbol{h}_{n}(\omega)$ to Eq.(6). The separated signal $\boldsymbol{u}(t)$ in the time domain can be obtained by Eq.(7).

\subsection{Scaling and Permutation Problem}

In the frequency domain, the indeterminacy of scaling and permutation occur at every frequency $\omega$ :

$$
H(\omega) G(\omega)=P(\omega) D(\omega)
$$

where $P(\omega)$ is a permutation matrix, which all elements of each column and row 0 except for one element with value 1 , and $D(\omega)=\operatorname{diag}\left[d_{1}(\omega), \cdots, d_{N}(\omega)\right]$ a diagonal matrix, of which elements $d_{n}(\omega)$ denote the scaling factors determined by the whitening. This means that not only the amplitude but also the phase are indeterminate. Therefore, the indeterminacy of permutation, the amplitude and the phase must be settled to get a meaningful signal $u(t)$ before inversely transforming $u(\omega, k)$ from the frequency to the time domain.

\subsection{Decomposed Spectrum and Scaling Ad- justment}

Hereafter we denote subscript $\hat{i}$ instead of $n$ to clarify the correspondence to $i$-th source. A decomposed spectrum $v_{\hat{i}}(\omega, k)=\left[v_{\hat{i} 1}(\omega, k), \cdots ; v_{\hat{i} J}(\omega, k)\right]^{T}$ is first introduced by Murata et al. as follows [7].

$$
\boldsymbol{v}_{\hat{i}}(\omega, k)=H^{-1}(\omega)\left[0, \cdots, 0, u_{\hat{i}}(\omega, k), 0, \cdots, 0\right]^{T}
$$

Then we have proved that the decomposed spectrum is uniquely expressed as a product of $i$-th source $s_{i}(\omega, k)$ and $g_{j i}(\omega)$ from the $i$-th source to the $j$-th microphone as folows $[12,13]$.

$$
v_{\hat{i}}(\omega, k)=\left[g_{1 i}(\omega) s_{i}(\omega, k), \cdots, g_{J i}(\omega) s_{i}(\omega, k)\right]^{T}
$$

This means that the decomposed spectrum $v_{\hat{i}}$ is the observation of $i$-th source $s_{i}(\omega, k)$ through $j$-th microphone.It is also clarified that every decomposed spectrum has no ambiguity of scaling in that the scaling factor is a transfer function itself; while the scaling factor $d_{n}(\omega)$ for the separated spectrum $u_{n}(\omega, k)$ varies arbitrarily, depending on the whitening result at individual frequency bin.

\section{A Solution of Permutation Problem and Target Selection}

\subsection{Permutation Correction}

We assume that the source $s_{i}(t)$ is closer to $i$-th microphone than to the others. From this assumption, a gain and a phase inequality on the transfer functions obtained, respectively, by

$$
\left|g_{i i}(\omega)\right|>\left|g_{j i}(\omega)\right|, \angle g_{i i}(\omega)>\angle g_{j i}(\omega) \text {. }
$$

From the above relations, we adopt the estimated spectrum of the source $s_{i}(\omega, k)$ as $g_{i i}(\omega) s_{i}(\omega, k)$, since it may be less affected by ambient noise. To do this, we calculate the absolute value of every component in $v_{\hat{i}}(\omega, k)$ and compare them to obtain the maximum value, since every component contains the same $s_{i}(\omega, k)$ and differs from the others by its transfer function.

Therefore, if $\left|v_{\hat{i} j}(\omega, k)\right|$ takes the maximum value at $j=i$, the number $i$ indicates the one corresponding to the source $s_{i}(\omega, k)$ and $v_{\hat{i}, j=i}(\omega, k)$ becomes the estimate of $s_{i}(\omega, k)$. Therefore a permutation correction rule are formulated with respect to the gain inequality:

$$
i=\arg \max _{j}\left|v_{\hat{i} j}(\omega, k)\right| \text {. }
$$

Similarly, another permutation correction rule is derived from the phase inequality:

$$
i=\arg \max _{j} \angle v_{\hat{i} j}(\omega, k) .
$$

\subsection{Target Source Estimation}

There still remains a target source selection problem even if the scaling and permutation problem can be settled. After permutation correction using Eq.(16) or Eq.(17), the separated spectrum $y_{i}(\omega, k)$ can be expressed as

$$
y_{i}(\omega, k)=g_{i i}(\omega) s_{i}(\omega, k) .
$$

This shows that $y_{i}(\omega, k)$ is an estimate of $s_{i}(\omega, k)$. If we know the target source number $i$ or the target source location in advance, we can extract the target source easily as

$$
y^{*}(\omega, k)=y_{i}(\omega, k) .
$$

The next section gives another selecting method in the case where the location of human speech is not known.

\subsection{Target Speech Selection}

We assume that one source is human speech and the others are noises, but we do not know the location of human speech. Human speech is usually larger in non-Gaussianity than noises. The FastICA $[5,6]$ proposed by Hyvärinen generates the separated signal in order of large non-Gaussianity. Under the above situation, therefore, the separated spectra for the speech are yielded at most frequencies to the first output channel. 


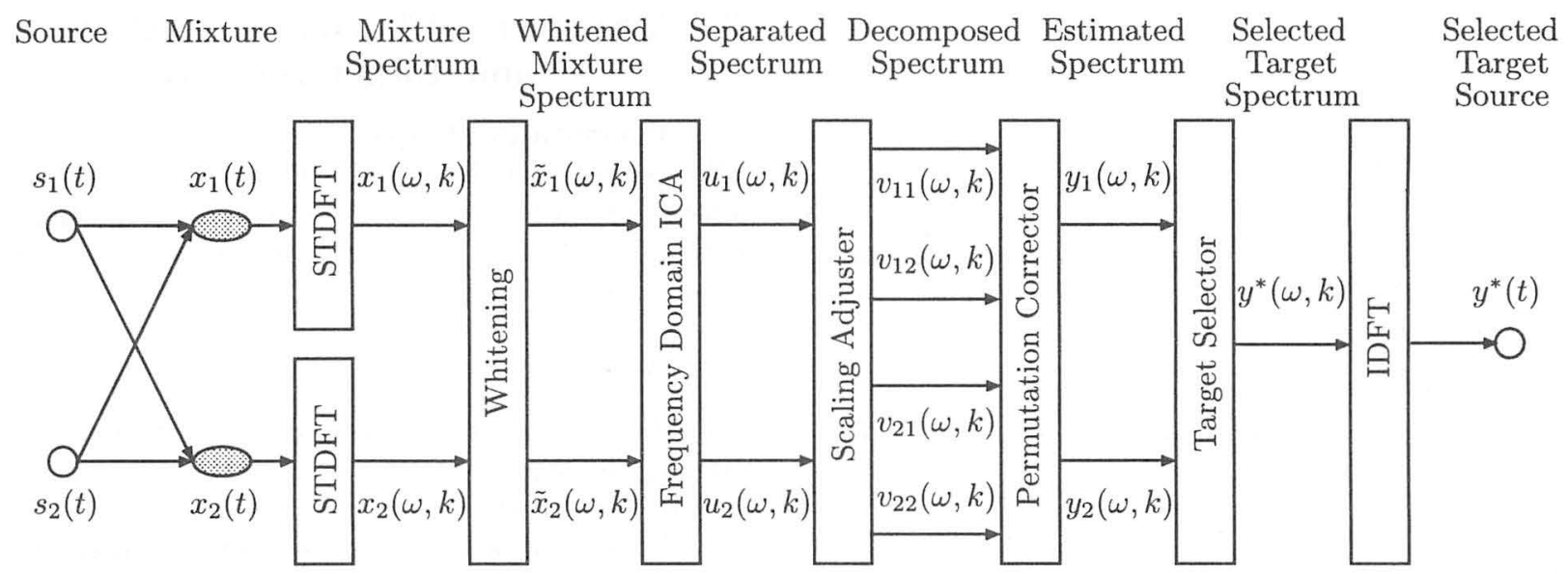

Fig. 2: Permutation corrector and target selector

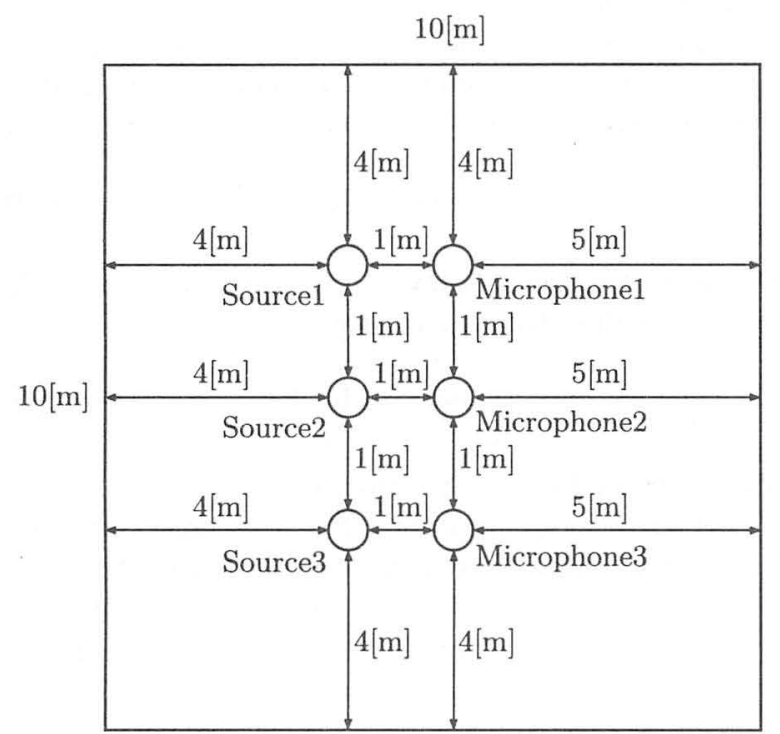

Fig. 3: Locations of sources and microphones in the virtual room

In other word, the first output channel gives the spectral estimate for the speech at the highest probability. Fig. 2 shows the procedure of our proposed method with two-source and two-microphone.

\section{Simulation}

In order to verify our proposals, several simulations were carried out. Source 1 was a speech signal [14], Source 2 was a roaring train noise recorded at a station premises [15] and Source 3 was a stationary noise [15]. In a virtual room $(L 10[m] \times W 10[m] \times H 10[m])$, the sound sources and the microphones are located at $1[\mathrm{~m}]$ height above the floor as in Fig.3. The impluse responce from the sources to the microphones were cal-
Table 1: Signal to Noise Ratio

\begin{tabular}{c|c|c|c}
\hline & Mixture & FastICA & Proposed \\
\hline SNR & $4.74[\mathrm{~dB}]$ & $5.05[\mathrm{~dB}]$ & $22.98[\mathrm{~dB}]$ \\
\hline
\end{tabular}

culated through MATLAB cade of BSS'99 Synthetic Benchmarks [16] and were used to generate 20 sets of convolved signals.

The convoluved signals were sampled at a rate of $8000[\mathrm{~Hz}]$ with $16[\mathrm{bit}]$ resolution. The sampled data were windowed by the Hamming window with a frame length $128[\mathrm{~ms}]$ and a frame period $32[\mathrm{~ms}]$. In the FastICA aigorithm, the weight were initialized by complex random values such that $\left\|\boldsymbol{h}_{n}(\omega)\right\|=1$, the nonlinear function was specified as

$$
f\left(\left|u_{n}(\omega, k)\right|^{2}\right)=1-2 /\left(e^{2\left|u_{n}(\omega, k)\right|^{2}}+1\right) .
$$

The algorithm was repeated until the convergence criterion

$$
\left|\overline{\boldsymbol{h}}_{n, \text { old }}^{T}(\omega) \boldsymbol{h}_{n, \text { new }}(\omega)\right|>0.999999
$$

was satisfied.

Table 1 shows the averaged SN ratio for the 20 data sets. From this results, it is found that the proposed method gives much better SN ratio than the FastICA.

Fig.4 shows the waveformes, (a) is sound sources, (b) is Mixture signals recorded by microphones, (c) is restored signals by the FastICA and (d) is restored signals by our proposed method. From the waveforms, the restored signals by the FastICA are found to be quite unsimilar to original source signals without the scaling and permutation problem resolved. The restored signals in the case of proposed are quite similar to the original sources. It is clarified that our proposed method outforms the FastICA. 


\section{Conclutions}

Our previously proposed permutation correction for the two-source and two-microphone cases has been extended for the multi-source and multi-microphone cases, where the decomposed spectrum is also shown to be a product of source and transfer function. Based on this fact, we have proposed a solution of permutation problem inherent in frequency domain ICA for the multi-source and multi-microphone cases. Furthermore, we have also shown how to select the human speech among the separated signals.

\section{References}

[1] A. J. Bell and T. J. Sejnowski: An information maximization approach to blind separation and blind deconvolution, Neural Computation, Vol. 7, No. 6, pp. 1129-1159, 1995.

[2] A. Cichocki and S. Amari: Adaptive blind signal and image processing, Learning algorithm and applications, John Wiley \& Sons, Ltd, 2002.

[3] S. Amari: Natural gradient works efficiently in learning, Neural Computation, Vol. 10, pp. 251276, 1998.

[4] T. W. Lee, M. Girolami and T. J. Sejnowski: Independent component analysis using an extended informax algorithm for mixed subgaussian and supergaussian sources, Neural Computation, Vol. 11, No. 2, pp. 417-441, 1999.

[5] A. Hyvärinen and E. Oja: Independent Component Analysis, Algorithms and applications, Neural Networks, Vol. 13, No. 4-5, pp. 411-430, 2000.

[6] E. Bingham and A. Hyvärinen: A fast fixed-point algorithm for independent component analysis for complex valued signals, Int. J. of Neural Systems, Vol. 10, No. 1, pp. 1-8, 2000.

[7] N. Murata, S. Ikeda and A. Ziehe: An approach to blind source separation based on temporal structure of speech signals, Neurocomputing, Vol. 41, Issue 1-4, pp. 1-24, 2001.

[8] S. Ding, M. Otsuka, N. Ashizawa, T. Niitsuma and K. Sugai: Blind source separation of real-world acoustic signals based on ICA in time-frequencydomain, IEICE Technical Report, EA2001-1, pp. $1-8,2001$.

[9] H. Saruwatari, S. Kurita, K. Takeda, F. Itakura, T. Nishikawa and K. Shikano: Blind source separation combining independent component analysis and beamforming, EURASIP Journal on Applied Signal Processing, Vol.2003, No.11, pp.1135-1146, 2003.
[10] H. Sawada, R. Mukai, S. Araki and S. Makino: A robust and precise method for solving the permutation problem of frequency-domain blind source separation, 4th International Symposium on Independent Component Analysis and Blind Signal Separation (ICA2003), pp. 505-510, 2003.

[11] K. Kaneda, K. Koya and H. Gotanda: Permutation resolution based on entropy of split spectrum; IEICE Trans., Vol. J87-A, No. 7, pp. 1065-1069, 2004. (in Japanese)

[12] H. Gotanda, K. Nobu, T. Koya, K. Kaneda, T. Ishibashi and N. Haratani: Permutation correction and speech extraction based on split spectrum through FastICA, 4th International Symposium on Independent Component Analysis and Blind Signal Separation (ICA2003), pp. 379-384, 2003.

[13] T. Ishibashi, K. Inoue, T. Koya, H. Gotanda and K. Kumamaru: Blind source separation without permutation and scaling indeterminacy, SICE Annual Conference 2004, pp. 2298-2301, 2004.

[14] Acoustical Society of Japan: ASJ continuous speech corpus japanese newspaper article sentences, JNAS Vols.1-16, 1997.

[15] NTT Advanced Technology Corporation: Ambient noise database for telephonometry 1996, 1996.

[16] http://sound.media.mit.edu/ica-bench/ 

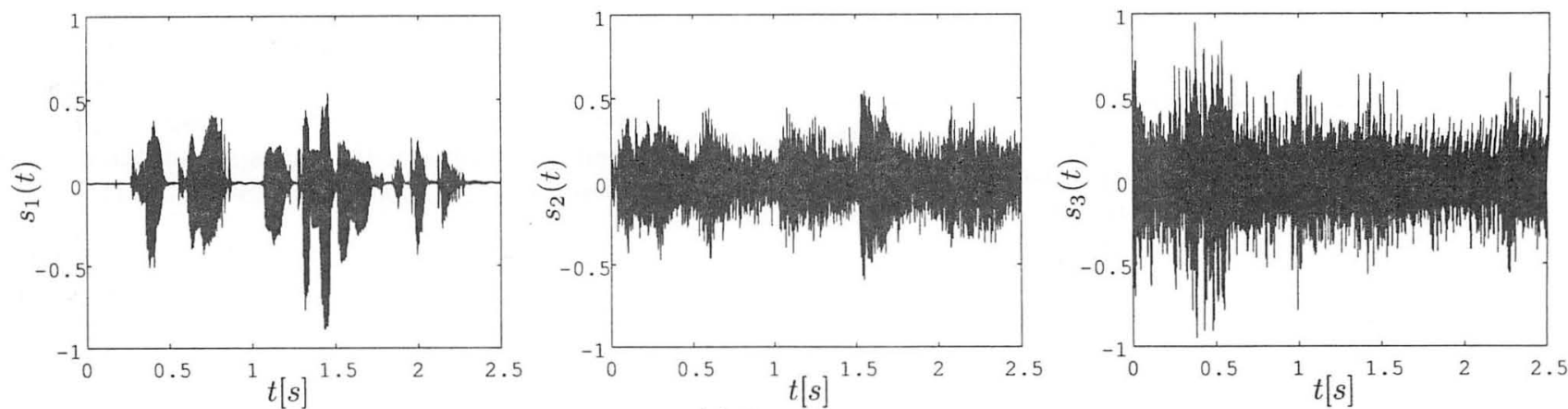

(a) Sound sources
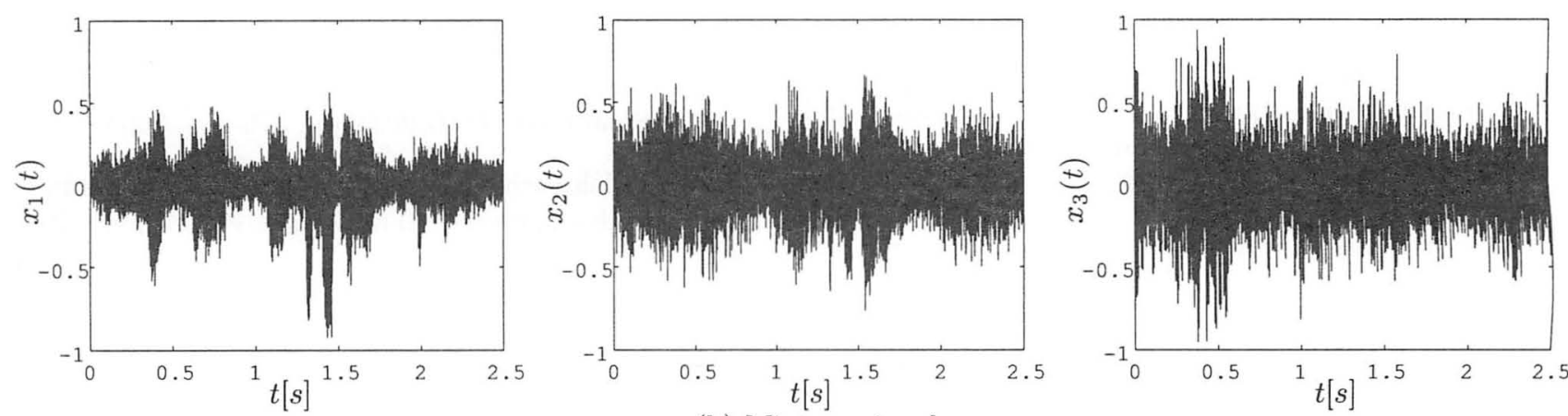

(b) Mixture signals
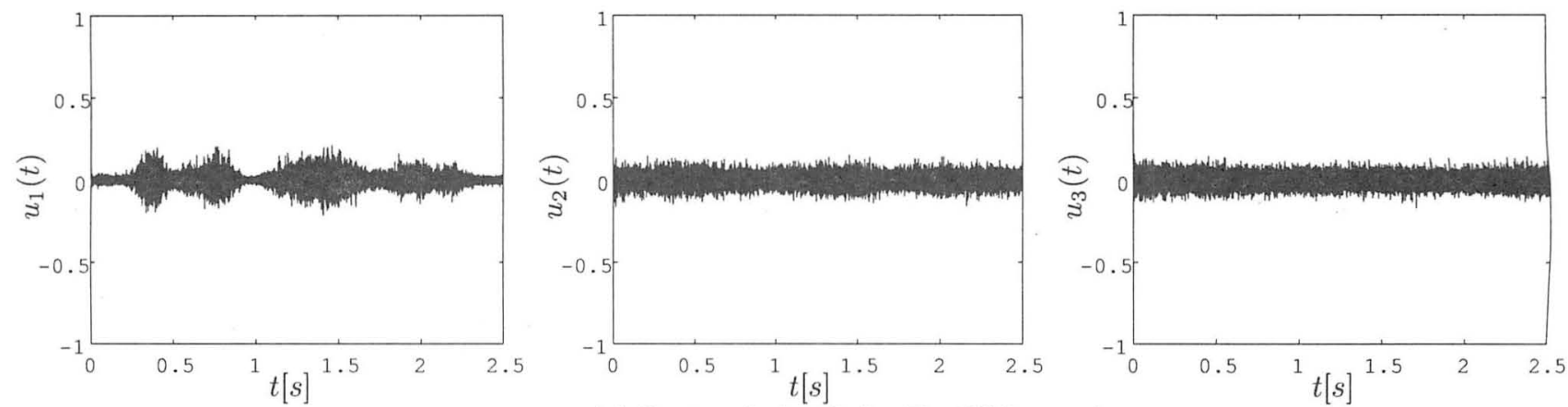

(c) Restored signals by FastICA
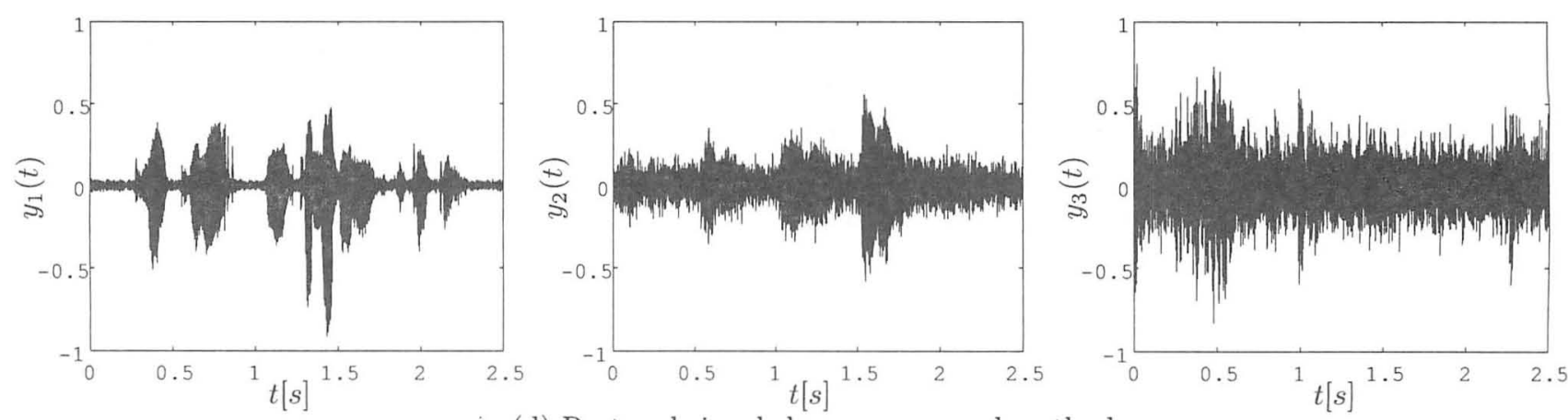

(d) Restored signals by our proposed method

Fig. 4: Experimental results: (a)Sources, (b) Mixture signals recorded by microphones, (c) Restored signals by FastICA, (d) Restored signals by our proposed method. 